\title{
Analysis of cesarean section rates using Robson Ten Group Classification System in a tertiary teaching hospital, Addis Ababa, Ethiopia: a cross- sectional study
}

Ferid A. Abubeker ( $\square$ ferid.abas@sphmmc.edu.et)

Saint Paul's Hospital Millennium Medical College https://orcid.org/0000-0002-2624-544X

Biruck Gashawbeza

Saint Paul's Hospital Millennium Medical College

Thomas Mekuria Gebre

Saint Paul's Hospital Millennium Medical College

Mekitie Wondafrash

Saint Paul's Hospital Millennium Medical College

Alula M. Teklu

MERQ Consultancy PLC

Demis Degu

Saint Paul's Hospital Millennium Medical College

Delayehu Bekele

Saint Paul's Hospital Millennium Medical College

Research article

Keywords: Cesarean section, Robson classification, Vaginal delivery, Tertiary level facility, Low income countries

Posted Date: December 16th, 2020

DOl: https://doi.org/10.21203/rs.3.rs-64829/v3

License: (c) (i) This work is licensed under a Creative Commons Attribution 4.0 International License. Read Full License

Version of Record: A version of this preprint was published on December 9th, 2020. See the published version at https://doi.org/10.1186/s12884-020-03474-x. 


\section{Abstract}

Background Cesarean section (CS) is an important indicator of access to, and quality of maternal health services. The World Health Organization recommends the Robson ten group classification system as a global standard for assessing, monitoring and comparing CS rates at all levels. This study aimed to assess the rate of CS and perform an analysis based on Robson classification system.

Methods A facility-based cross-sectional study was conducted at a tertiary hospital in Addis Ababa, Ethiopia. Data were collected from medical charts of all women who delivered from January-June 2018. The overall CS rate was calculated then women were categorized into one of the ten Robson groups. Relative size of each group, contribution of each group to the overall CS rate, and CS rate within each group were calculated.

Results A total of 4,200 deliveries were analyzed. Of these 1,459 (34.7\%) were CS. The largest contributors to the overall CS rate were Group 10 (19.1\%), Group 2 (18.3\%), Group 5 (17.1\%), and Group 4 (15.8\%). There was also a high rate of pre-labor CS in Group 2, Group 4, and Group 10.

Conclusion Through implementation of the Robson ten group classification system, we identified the contribution of each group to the overall CS rate as well as the CS rate within each group. Group 10 was the leading contributor to the overall CS rate. This study also revealed a high rate of CS among low-risk groups. These target groups require more in-depth analysis to identify possible modifiable factors and to apply specific interventions to reduce the CS rate. Evaluation of existing management protocols and further studies into indications of CS and outcomes are needed to design tailored strategies and improve outcomes.

\section{Background}

In 1985 the World Health Organization (WHO) stated a cesarean section (CS) rate higher than $10-15 \%$ is not justified for any region [1]. Thirty years after the publication of the WHO guidelines, there is no consensus about the optimal CS rate and appropriate interpretation of this indicator remains a topic of debate. More recent efforts to determine the optimal cs rate also had limitations due to lack of external validity and confounders $[2,3]$.

Despite its limitations, the proportion of cesarean section (CS) at a population level is an important indicator of access to, and quality of maternal health services offered in a country [4]. In 2016 the national population-based CS rare for Ethiopia was about 3\%, a figure far below the WHO optimum range of $10-15 \%$. The sub-national regional figures varied widely ranging from $25 \%$ in the capital, Addis Ababa, to less than $1 \%$ in more rural regions. Furthermore, the overall CS rates differ significantly between different institutions [5].

Several reasons can explain variations in institutional rates of CS. These include the inherent differences in patient characteristics, type of institution and available resources. In addition, institutional differences 
in obstetric practice and pregnancy and labor management protocols can account for this variation [6]. Therefore, population-based CS rates should not be considered as recommended targets at facility level. Indeed, systems designed to monitor cesarean section rates at facilities should take into account these differences. CS rates should no longer be thought of being too high or too low but rather whether or not they are appropriate. Thus, CS should only be conducted based on medical indications, and efforts should be directed towards improving access to all women in need rather than striving to achieve an arbitrary rate $[6,7]$.

To this end, policymakers, program managers, clinicians, and administrators need a standardized and internationally accepted classification system to monitor and compare CS rates in a meaningful, reliable and action-oriented manner [8]. A systematic review of existing CS classification system conducted in 2011 identified 27 different classification systems of which Robson's Ten Group Classification System (RTGCS) was found to be the best option [9].

The Robson classification system classifies all deliveries into ten mutually exclusive and totally inclusive groups based on a set of predefined obstetric parameters. These include parity, previous CS, onset of labor, fetal presentation, number of fetuses and gestational age (Table 1). Each Robson group is further analyzed to assess its relative size to the obstetric population, its contribution to the overall CS rate, and the CS rate within the group [8]. The classification system is simple to use and enables auditing and analyzing CS rates as it is based on routinely documented obstetrics characteristics of individual woman without relying on the indication for CS.

Effective application of the RTGCS offers several advantages. It allows identification of the Robson groups that make significant contributions to the overall CS rate. This is a crucial step in the audit process as interventions that make even small changes to the CS rate within these target groups can bring about significant changes to the overall CS rate $[7,8]$. The system is easily reproducible and offers a standardized comparison method within a particular institution over time or between institutions at a national, regional or global level [6-9]. In addition, RTGCS can inform the impact of interventions at both institutional and national levels by providing a benchmark and analyzing the trends in the overall and group-specific CS rates over time [10].

Cognizant of its advantages and simplicity, the WHO and the International Federation of Gynecology and Obstetrics (FIGO) recommend the Robson classification system as a global standard for assessing, monitoring and comparing CS rates among nations and within institution over time, and between institutions, regardless of their level of complexity $[6,11,12]$.

\section{Table 1. Robson ten group delivery classification system}




\section{Groups Description}

Group Nulliparous, single cephalic, $\geq 37$ weeks, in spontaneous labor

1

Group Nulliparous, single cephalic, $\geq 37$ weeks, induced or CS before labor

2 2a- Nulliparous, singleton, cephalic, $\geq 37$ weeks' gestation, induced labor.

2b- Nulliparous, singleton, cephalic, $\geq 37$ weeks' gestation, cesarean section before labor.

Group Multiparous (excluding previous cesarean section), singleton, cephalic, $\geq 37$

3 weeks' gestation, in spontaneous labor.

Group Multiparous without a previous uterine scar, with singleton, cephalic

4 pregnancy, $\geq 37$ weeks' gestation, induced or cesarean section before labor.

4a- Multiparous without a previous uterine scar, with singleton, cephalic pregnancy, $\geq 37$ weeks' gestation, induced labor.

4b- Multiparous without a previous uterine scar, with singleton, cephalic pregnancy, $\geq 37$ weeks' gestation, cesarean section before labor.

Group Previous cesarean section, singleton, cephalic, $\geq 37$ weeks' gestation. 5

Group All nulliparous with a single breech.

6

Group All multiparous with a single breech (including previous cesarean section). 7

Group All multiple pregnancies (including previous cesarean section).

8

Group All women with a single pregnancy in transverse or oblique lie (including those

9 with previous cesarean section).

Group All singleton, cephalic, <37 weeks' gestation pregnancies (including previous 10 cesarean section).

Many obstetric units around the world have successfully utilized RTGCS. Application of the classification system in different institutions across the world have yielded similar results, although some had significant differences [13-16]. The aim of this study was thus to assess the rate of CS in our institution and perform an analysis based on Robson ten group classification system.

\section{Methods}

\section{Study design, setting, and participants}

This was a cross-sectional study conducted at Saint Paul's Hospital Millennium Medical College (SPHMMC) in Addis Ababa, Ethiopia. SPHMMC is a tertiary care facility conducting close to 10,000 
deliveries per annum. It is also a public teaching hospital and mainly serves as a referral center for highrisk cases.

The study population included all women who gave birth from January to June 2018. We excluded laparotomy done for uterine rupture and deliveries before fetal viability. In the Ethiopian context, viability is considered after gestational age of 28 weeks or birth weight $\geq 1,000 \mathrm{~g}$, if gestational age is unknown [17].

\section{Data source and variables}

Data were collected by trained data collectors using a structured data extraction template on Open Data Kit for an android platform and saved on a central server. Medical charts were reviewed to collect relevant obstetric information. This includes past obstetric history (parity and previous CS), onset of labor (spontaneous, induced, or CS before labor), fetal presentation or lie (cephalic, breech, transverse or oblique), number of fetuses (single or multiple), mode of delivery (vaginal or CS), and gestational age (term or preterm). Gestational age was calculated either from menstrual date or obstetric ultrasound done before 24 weeks of pregnancy. For cases with no gestational age milestone, we used birth weight as a proxy indicator of gestational age. Birth weight $<2,500 \mathrm{~g}$ was considered preterm and birth weight $\geq$ $2,500 \mathrm{~g}$ was considered term [18]. This strategy has been employed in other studies conducted in similar settings $[14,19,20]$.

\section{Data processing and analysis}

Data was exported to and analyzed using IBM SPSS Statistics for Windows, version 20 (IBM Corp., Armonk, N.Y., USA). The overall CS rate at the institution was calculated first. We coded all abstracted data and women were categorized into one of the ten Robson groups. For each group, size relative to the entire obstetric population, contribution to the overall CS rate, and CS rate within the group were calculated.

\section{Results}

Over the six-month period, a total of 4,226 women presented for labor and delivery. Five patients were excluded for uterine rupture and 21 were excluded with pre-viable deliveries. Thus, a total of 4,200 deliveries were analyzed. The mean age of participants was 26.4 years (SD 4.7). The rate of CS was $34.7 \%$ (Table 2).

Table 2. Characteristics of women who gave birth at SPHMMC, Addis Ababa, Ethiopia, January-June 2018 


\begin{tabular}{|c|c|c|}
\hline Characteristics & Frequency (n) & Percentage (\%) \\
\hline $\begin{array}{l}\text { Age (years) } \\
<20 \\
20-34 \\
\geq 35 \\
\end{array}$ & $\begin{array}{c}174 \\
3,719 \\
307\end{array}$ & $\begin{array}{c}4.2 \\
88.5 \\
7.3 \\
\end{array}$ \\
\hline $\begin{array}{l}\text { Parity } \\
0 \\
1-4 \\
\geq 5\end{array}$ & $\begin{array}{l}1,980 \\
2,147 \\
\quad 73\end{array}$ & $\begin{array}{c}47.1 \\
51.1 \\
1.8\end{array}$ \\
\hline $\begin{array}{l}\text { Gestational Age* } \\
<37 \text { weeks } \\
\geq 37 \text { weeks }\end{array}$ & $\begin{array}{c}821 \\
3,379\end{array}$ & $\begin{array}{l}19.5 \\
80.5\end{array}$ \\
\hline $\begin{array}{l}\text { Onset of labor } \\
\text { Spontaneous } \\
\text { Induced } \\
\text { CS before onset of labor }\end{array}$ & $\begin{array}{l}2,787 \\
346 \\
1,067\end{array}$ & $\begin{array}{l}66.4 \\
8.2 \\
25.4\end{array}$ \\
\hline $\begin{array}{l}\text { Fetal presentation/lie } \\
\text { Cephalic } \\
\text { Breech } \\
\text { Transverse/Oblique }\end{array}$ & $\begin{array}{c}3,967 \\
219 \\
14\end{array}$ & $\begin{array}{l}94.5 \\
5.2 \\
0.3\end{array}$ \\
\hline $\begin{array}{l}\text { Number of fetus } \\
\text { Single } \\
\text { Multiple }\end{array}$ & $\begin{array}{c}4,024 \\
176\end{array}$ & $\begin{array}{c}95.8 \\
4.2\end{array}$ \\
\hline $\begin{array}{l}\text { Mode of delivery } \\
\text { Vaginal delivery } \\
\text { Cesarean section }\end{array}$ & $\begin{array}{l}2,741 \\
1,459\end{array}$ & $\begin{array}{l}65.3 \\
34.7 \\
\end{array}$ \\
\hline $\begin{array}{l}\text { Mode of operation } \\
\text { Emergency } \\
\text { Scheduled }\end{array}$ & $\begin{array}{l}935 \\
524\end{array}$ & $\begin{array}{l}64.1 \\
35.9\end{array}$ \\
\hline $\begin{array}{l}\text { Fetal outcome** } \\
\text { Live birth } \\
\text { Stillbirth }\end{array}$ & $\begin{array}{c}4,138 \\
240\end{array}$ & $\begin{array}{c}94.5 \\
5.5\end{array}$ \\
\hline $\begin{array}{l}\text { Birth weight (gram)** } \\
<1,000 \\
1,000-2,499 \\
2,500-3,999 \\
\geq 4,000\end{array}$ & $\begin{array}{c}30 \\
953 \\
3,234 \\
161\end{array}$ & $\begin{array}{c}0.7 \\
21.8 \\
73.8 \\
3.7\end{array}$ \\
\hline
\end{tabular}

*For 839 women with unknown gestational age, birth weight was used as a proxy indicator, 177 newborns had birth weight $<2,500 \mathrm{~g}$ and were considered $<37$ weeks whereas 662 newborns had birth weight $\geq 2,500 \mathrm{~g}$ and were considered $\geq 37$ weeks.

**Rate calculated per total number of babies born.

Women in Group 1 (nulliparous women with single cephalic pregnancy at term in spontaneous labor) made the largest contribution to the obstetric population accounting for $26.7 \%$ of all deliveries. This was followed by Group 3 (multiparous women with single cephalic pregnancy at term in spontaneous labor without previous CS) and Group 10 (all women with single cephalic pregnancy before term, including those with previous CS) which accounted for $22.2 \%$ and $15.9 \%$ respectively. Group 5 (all multiparous women with single cephalic pregnancy at term and at least one previous uterine scar) was the fourth largest group accounting for $9.5 \%$ of the obstetric population (Table 3 ). 
The largest contributors to the overall CS rate were Group 10 (19.1\%), Group 2 (nulliparous women with single cephalic pregnancy at term who either had an induction of labor or CS before the onset of labor) (18.3\%), Group 5 (17.1\%), and Group 4 (multiparous women with single cephalic pregnancy at term without previous CS who either had an induction of labor or CS before the onset of labor) (15.8\%). These 4 groups contributed for about $70 \%$ of all cesarean deliveries (Table 3 ).

Table 3: - Proportion of each Robson groups, CS rate in each group, and their relative and absolute contribution to overall CS rate at SPHMMC, Addis Ababa, Ethiopia, January-June 2018

\begin{tabular}{|c|c|c|c|c|c|c|}
\hline $\begin{array}{l}\text { Robson } \\
\text { Group }\end{array}$ & $\begin{array}{l}\text { Number } \\
\text { of CS in } \\
\text { group }\end{array}$ & $\begin{array}{l}\text { Total } \\
\text { number of } \\
\text { women in } \\
\text { group }\end{array}$ & $\begin{array}{l}\text { Group } \\
\text { size } \\
(\%)^{1}\end{array}$ & $\begin{array}{l}\text { Group } \\
\text { CS } \\
\text { rate } \\
(\%)^{2}\end{array}$ & $\begin{array}{l}\text { Absolute group } \\
\text { contribution to } \\
\text { overall CS rate } \\
(\%)^{3}\end{array}$ & $\begin{array}{l}\text { Relative group } \\
\text { contribution to } \\
\text { overall CS rate } \\
(\%)^{4}\end{array}$ \\
\hline $\begin{array}{l}\text { Group } \\
1\end{array}$ & 156 & 1,121 & 26.7 & 13.9 & 3.7 & 10.7 \\
\hline $\begin{array}{l}\text { Group } \\
2\end{array}$ & 267 & 368 & 8.8 & 72.6 & 6.4 & 18.3 \\
\hline $2 \mathrm{a}$ & 34 & 135 & 3.2 & 25.2 & 0.8 & 2.3 \\
\hline $2 b$ & 233 & 233 & 5.6 & 100.0 & 5.6 & 16.0 \\
\hline $\begin{array}{l}\text { Group } \\
3\end{array}$ & 66 & 934 & 22.2 & 7.1 & 1.6 & 4.5 \\
\hline $\begin{array}{l}\text { Group } \\
4\end{array}$ & 231 & 327 & 7.8 & 70.6 & 5.5 & 15.8 \\
\hline $4 a$ & 6 & 102 & 2.4 & 5.9 & 0.1 & 0.4 \\
\hline $4 b$ & 225 & 225 & 5.4 & 100.0 & 5.4 & 15.4 \\
\hline $\begin{array}{l}\text { Group } \\
5\end{array}$ & 249 & 397 & 9.5 & 62.7 & 5.9 & 17.1 \\
\hline $\begin{array}{l}\text { Group } \\
6\end{array}$ & 48 & 94 & 2.2 & 51.1 & 1.1 & 3.3 \\
\hline $\begin{array}{l}\text { Group } \\
7\end{array}$ & 56 & 101 & 2.4 & 55.4 & 1.3 & 3.8 \\
\hline $\begin{array}{l}\text { Group } \\
8\end{array}$ & 95 & 176 & 4.2 & 54.0 & 2.3 & 6.5 \\
\hline $\begin{array}{l}\text { Group } \\
9\end{array}$ & 13 & 13 & 0.3 & 100.0 & 0.3 & 0.9 \\
\hline $\begin{array}{l}\text { Group } \\
10\end{array}$ & 278 & 669 & 15.9 & 41.6 & 6.6 & 19.1 \\
\hline Total & 1,459 & 4,200 & 100 & 34.7 & 34.7 & 100 \\
\hline
\end{tabular}

${ }^{1}$ Group size $(\%)=\mathrm{n}$ of women in the group/total $\mathrm{N}$ women delivered in the hospital $\times 100$

${ }^{2}$ Group CS rate $(\%)=\mathrm{n}$ of CS in the group/total $\mathrm{N}$ of women in the group $\times 100$

${ }^{3}$ Absolute group contribution $(\%)=\mathrm{n}$ of CS in the group/total $\mathrm{N}$ of women delivered in the hospital $\times 100$

${ }^{4}$ Relative group contribution $(\%)=\mathrm{n}$ of CS in the group/total $\mathrm{N}$ of CS in the hospital $\times 100$

Further subgroup analysis of women in Group 10 showed 323 (48.3\%) had spontaneous onset of labor while 107 (16\%) were induced. The remaining 239 (35.7\%) underwent CS before the onset of labor. A similar analysis showed 233 (63.3\%) in Group 2 and 225 (68.8\%) in Group 4 had pre-labor CS. 
Group 3 accounted for $4.5 \%$ of the overall CS rate and $7.1 \%$ of women within this group had CS. Group 6 and Group 7 represent nulliparous and multiparous women with breech presentation respectively. Together these two groups contributed $7.1 \%$ to the overall CS whereas the CS rate within each group was about $50 \%$ (Table 3 ).

\section{Discussion}

Cesarean section is a key intervention to decrease maternal and neonatal morbidity and mortality. It is also one of the best indicators of the quality of maternal health services [4]. Despite its proven benefits, it has associated complications such as infection, bleeding, anesthetic accidents and even death. Future pregnancies can also be complicated by spontaneous preterm birth, uterine rupture, and abnormal placentation. These risks are higher for women in resource-limited settings with poor access to comprehensive obstetric care [6,21].

Thus, to optimize outcomes, facilities should initiate a detailed and rigorous assessment of their practice vis-à-vis the case mix of obstetric population they serve. The Robson ten group classification system enables institution-specific monitoring and auditing and can be a powerful tool to inform practice across different settings $[6,8]$.

In this study, we implemented the RTGCS and assessed the proportion of each group in the obstetric population, the contribution of CS in each group to the overall CS rate and the CS rate within each group.

In our study, Group 1 and Group 3 represented the two largest groups presenting for labor and delivery. This finding is consistent with a study done in India where Group 1 and Group 3 contributed to 24.2\% and $19.4 \%$ of all deliveries respectively [16]. Similarly, studies done in Brazil, Italy, and Tanzania showed Group 3 and Group 1 were the two most represented obstetric groups [13-15].

Group 10 was found to be the third-largest obstetric group. The contribution of this group to the overall CS rate depends on its size [7]. As such, Group 10 made the highest contribution to the CS rate accounting for nearly one in five CS deliveries. This is in sharp contrast with a study done at a university hospital in Eastern Ethiopia, where Group 10 was the $6^{\text {th }}$ place contributor to the overall CS rate, accounting for $6 \%$ of CS deliveries [19]. This variation can be explained by the significant difference in the obstetric population served by the two facilities. Our study was done in a tertiary referral hospital with a dedicated maternal-fetal medicine unit. A significant proportion of care is given to mothers with major obstetric and medical comorbidities who may require interventions, increasing the likelihood of iatrogenic prematurity. This can account for the higher proportion of Group 10 and its contribution to the CS rate in our setting. In fact, our finding is consistent with studies conducted in other tertiary care facilities $[7,8]$. A study from a tertiary unit in Italy also showed Group 10 was the second largest contributor to the CS rate [15].

About $20 \%$ of women in our study did not have a milestone to ascertain gestational age. This is not unusual as the national guideline does not include routine dating ultrasound during antenatal care follow 
up [22] and has not yet adopted the WHO recommendation of at least one ultrasound scan before 24 weeks of gestation [23]. In this study, birth weight was used as an indirect estimate of gestational age for those women we could not ascertain gestational age. This adaptation has also been used in other studies that implemented the RTGCS in low-resource settings. For example, Tura AK et al [19] and Schantz $C$ et al [24] used birth weight of $\geq 2,500 \mathrm{~g}$ as proxy to term when data on gestational age was not available whereas other studies applied the method to their entire dataset and defined gestational age solely based on birth weight $[14,20,25]$.

Using neonatal birth weight as a proxy indicator of gestational age can however result in misclassification of growth-restricted newborns as preterm and potentially increase the relative proportion of Group 10. To test for this, we performed a separate analysis after excluding the 839 women with unknown gestational age. However, there was no change to the relative proportion of Group 10 and its contribution to the CS rate, and the group remained the third largest group and the leading contributor to the overall CS rate.

In addition, the CS rate within Group 10 was found to be $41 \%$, which indicates the possibility of a high rate of pre-labor CS (Table 3) [7]. Subgroup analysis shows one-third of women in this group underwent CS before the onset of labor. Further examination of the indications for CS can help us understand and design tailored interventions to reduce the CS rate in this group. This is especially relevant as interventions to reduce the CS rate within this particular group were found to be successful in other facilities [13].

Group 2 and Group 4 were also important contributors to the overall CS rate, accounting for one-third of CS deliveries. The CS rate within each group was also about 70\%. Existing evidence suggests a high prelabor CS rate at a particular institution if the CS rate within Group 2 and Group 4 is more than $35 \%$ and $20 \%$, respectively [7]. Subdividing these groups into induced labor and CS before labor provides useful information regarding the proportion of pre-labor CS and the success of induction (Table 3). This is particularly important as women in these groups are considered low risk. Our subgroup analysis showed a large proportion of women in both groups underwent pre-labor CS. This calls for further investigation of the indications for pre-labor CS. Similarly, a high rate of CS in these low risk groups was observed in high resource settings like Italy, Singapore and Brazil $[15,26,27]$. For instance, the CS rate within Group 2 and Group 4 was $82 \%$ and $62 \%$ respectively at a public hospital in Brazil [27].

Several studies across different settings identified Group 5 as the leading contributor to the CS rate [13, $14,16]$. In our study, Group 5 was the third-largest contributor to the overall CS rate and its relative size to the obstetric population was less than $10 \%$. These findings are suggestive of relatively low CS rate in the previous years [7]. Indeed, in 2016 the national institutional and population-based CS rates were $4 \%$ and $2.7 \%$ respectively [5].

Though the safety and long-term benefits of vaginal birth after cesarean (VBAC) are well established, 62\% of women in Group 5 underwent repeat CS (Table 3). Thus, there is a need to evaluate the proportion of women who were offered a trial of labor and the success rate of VBAC. This will enable the design and 
implementation of antenatal counseling strategies and labor management protocols, reducing the number of repeat CS.

The contribution of Group 3 to the overall CS rate was small. However, the high rate of CS within this group is an alarming finding. The group represents low-risk women and the CS rate within this group is not expected to be higher than $3 \%$. Auditing this group is an effective means to assess how an institution manages labor [7]. Thus, evaluation of labor management protocols in our institution is warranted.

Since the publication of the term breech trial, there is a global shift towards CS among women with breech presentation $[28,29]$. Consequently, several studies showed a high rate of CS in Group 6 and 7 [13, 15]. However, in our study, nearly half of breech presentations both in nulliparous and multiparous women were delivered vaginally (Table 3 ). This is similar to the $40 \%$ vaginal breech delivery observed in another teaching hospital in southwest Ethiopia [30]. A more liberal national and institutional protocols that allow assisted vaginal breech delivery in selected women can explain these observations [22]. Though we find this practice encouraging, further analysis should be done to assess maternal and perinatal outcomes among these groups.

The strengths of this study include the large sample size and availability of complete data for analysis. The results of this study can serve as baseline data to monitor trends of CS rate over time in our institution, as well as to compare our practice with that in other institutions.

This study also has some limitations. Our definition of fetal viability based on gestational age of 28 weeks or birth weight of $\geq 1,000 \mathrm{~g}$ may affect the rate of CS and the relative size of Robson's groups. This in turn can impact the generalizability of our findings to other countries. Findings from RTGCS are only a starting point and should be viewed as a means, not an end. We now have a clear insight about "who" is having CS but not "why" the CS is being performed. Crucial variables such as indications, maternal and perinatal outcomes, are not incorporated, limiting the extent to which conclusions can be drawn from our study.

\section{Conclusion}

We used the RTGCS to identify specific groups that contributed the most to the overall CS in our setting. Group 10 was the leading contributor to the overall CS rate. This study also revealed a high rate of CS among low-risk groups. These target groups require more in-depth analysis to identify possible modifiable factors and to apply specific interventions to reduce the CS rate. Evaluation of existing management protocols and further studies into indications of CS and outcomes in our setting are needed to design tailored strategies and improve outcomes.

\section{List Of Abbreviations}

CS: Cesarean Section 
FIGO: International Federation of Gynecology and Obstetrics

RTGCS: Robson's Ten Group Classification System

SPHMMC: Saint Paul's Hospital Millennium Medical College

VBAC: vaginal birth after cesarean

WHO: World Health Organization

\section{Declarations}

\section{Ethical approval and consent to participate}

The study was approved by the institutional review board of Saint Paul's Hospital Millennium Medical College. Permission to collect data was obtained from the Obstetrics and Gynecology department.

\section{Consent for publication}

Not applicable

\section{Availability of data and material}

All dataset used and/or analysed during the current study are available from the croosponding author on reasonable request.

\section{Competing interests}

The authors declare that they have no competing interests

\section{Funding}

This study was funded by Saint Paul's Hospital Millennium Medical College. The funder of the study had no role in study design, data collection, analysis, interpretation, or writing of the report.

\section{Authors' contribution}

DB and FAA conceptualized the study. FAA, BG, TG and AMT wrote the initial draft of the study. TG, AMT and DD oversaw the data collection. FAA and MW did the analysis. FAA, BG and DD wrote the first draft of the manuscript. DB and MW interpreted the data and provided critical revisions. All authors contributed to and approved the final version of the manuscript to be published.

\section{Acknowledgments}


We would like to acknowledge the Department of Obstetrics and Gynecology at Saint Paul's Hospital Millennium Medical College, Addis Ababa, Ethiopia.

The authors thank professor Sarah Prager for proofreading and editing the manuscript.

\section{References}

1. World Health Organization: Appropriate technology for birth. Lancet 1985, 2:436-437.

2. Ye J, Betrán AP, Guerrero Vela M, Souza JP, Zhang J: Searching for the optimal rate of medically necessary cesarean delivery. Birth 2014, 41(3):237-244.

3. Betran AP, Torloni MR, Zhang J, Ye J, Mikolajczyk R, Deneux-Tharaux C, Oladapo OT, Souza JP, Tunçalp Ö, Vogel JP: What is the optimal rate of caesarean section at population level? A systematic review of ecologic studies. Reproductive health 2015, 12(1):57.

4. WHO, UNFPA, UNICEF, AMDD: Monitoring emergency obstetric care: a handbook. Geneva: World Health Organization; 2009.

5. Ethiopian Public Health Institute, Federal Ministry of Health, Averting Maternal Death and Disability (AMDD): Ethiopian Emergency Obstetric and Newborn Care (EmONC) Assessment 2016; Final Report. Addis Ababa, Ethiopia Ethiopian Public Health Institute; 2017.

6. World Health Organization: WHO statement on caesarean section rates, vol. WHO/RHR/15.02. Geneva: World Health Organization (WHO/RHR/15.02); 2015.

7. Robson M, Hartigan L, Murphy M: Methods of achieving and maintaining an appropriate caesarean section rate. Best Pract Res Clin Obstet Gynaecol 2013, 27(2):297-308.

8. Robson MS: Classification of caesarean sections. Fetal Matern Med Rev 2001, 12(1):23-39.

9. Torloni MR, Betran AP, Souza JP, Widmer M, Allen T, Gulmezoglu M, Merialdi M: Classifications for cesarean section: a systematic review. PLoS One 2011, 6(1):e14566.

10. Betran AP, Vindevoghel N, Souza JP, Gulmezoglu AM, Torloni MR: A systematic review of the Robson classification for caesarean section: what works, doesn't work and how to improve it. PLoS One 2014, 9(6):e97769.

11. FIGO Working Group on Challenges in Care of Mothers and Infants during Labour and Delivery: Best practice advice on the 10-Group Classification System for cesarean deliveries. International Journal of Gynecology \& Obstetrics 2016, 135(2):232-233.

12. Visser G, Ayres-de-Campos D, Barnea E, de Bernis L, Di Renzo G, Vidarte M, Lloyd I, Nassar A, Nicholson W, Shah P: FIGO position paper: how to stop the caesarean section epidemic. The Lancet 2018, 392(10155):1286-1287.

13. Costa ML, Cecatti JG, Souza JP, Milanez HM, Gulmezoglu MA: Using a Caesarean Section Classification System based on characteristics of the population as a way of monitoring obstetric practice. Reprod Health 2010, 7:13. 
14. Litorp H, Kidanto HL, Nystrom L, Darj E, Essen B: Increasing caesarean section rates among low-risk groups: a panel study classifying deliveries according to Robson at a university hospital in Tanzania. BMC Pregnancy Childbirth 2013, 13:107.

15. Triunfo S, Ferrazzani S, Lanzone A, Scambia G: Identification of obstetric targets for reducing cesarean section rate using the Robson Ten Group Classification in a tertiary level hospital. Eur $\mathrm{J}$ Obstet Gynecol Reprod Biol 2015, 189:91-95.

16. Sah S, Goel R, Goel JK: Analysis of caesarean section rate according to Robson's criteria in tertiary care centre. International Journal of Reproduction, Contraception, Obstetrics and Gynecology 2018, 7(8):3060-3064.

17. Ethiopian Ministry of Health: Technical and Procedural Guidelines for Safe Abortion Services in Ethiopia, Second edn. Addis Ababa, Ethiopia: Federal Ministry of Health; 2013.

18. World Health Organization: Physical status: The use of and interpretation of anthropometry, Report of a WHO Expert Committee. Geneva: World Health Organization; 1995.

19. Tura AK, Pijpers O, de Man M, Cleveringa M, Koopmans I, Gure T, Stekelenburg J: Analysis of caesarean sections using Robson 10-group classification system in a university hospital in eastern Ethiopia: a cross-sectional study. BMJ open 2018, 8(4):e020520.

20. Hanson C, Betrán A, Opondo C, Mkumbo E, Manzi F, Mbaruku G, Schellenberg J: Trends in caesarean section rates between 2007 and 2013 in obstetric risk groups inspired by the Robson classification: results from population-based surveys in a low-resource setting. BJOG 2019, 126(6):690-700.

21. Harrison MS, Pasha O, Saleem S, Ali S, Chomba E, Carlo WA, Garces AL, Krebs NF, Hambidge KM, Goudar SS: A prospective study of maternal, fetal and neonatal outcomes in the setting of cesarean section in low-and middle-income countries. Acta Obstet Gynecol Scand 2017, 96(4):410-420.

22. Ethiopian Ministry of Health: Management protocol on selected obstetrics topics. Addis Ababa, Ethiopia: Federal Ministry of Health; 2010.

23. World Health Organization: WHO recommendations on antenatal care for a positive pregnancy experience: World Health Organization; 2016.

24. Schantz C, Ravit M, Traoré AB, Aboubakar M, Goyet S, de Loenzien M, Dumont A: Why are caesarean section rates so high in facilities in Mali and Benin? Sexual \& reproductive healthcare 2018, 16:10-14.

25. Tognon F, Borghero A, Putoto G, Maziku D, Torelli GF, Azzimonti G, Betran AP: Analysis of caesarean section and neonatal outcome using the Robson classification in a rural district hospital in Tanzania: an observational retrospective study. BMJ open 2019, 9(12).

26. Chong C, Su LL, Biswas A: Changing trends of cesarean section births by the Robson Ten Group Classification in a tertiary teaching hospital. Acta Obstet Gynecol Scand 2012, 91(12):1422-1427.

27. Bolognani CV, Reis LBdSM, Dias A, Calderon IdMP: Robson 10-groups classification system to access C-section in two public hospitals of the Federal District/Brazil. PLoS One 2018, 13(2):e0192997.

28. Hannah ME, Hannah WJ, Hewson SA, Hodnett ED, Saigal S, Willan AR, Collaborative TBT: Planned caesarean section versus planned vaginal birth for breech presentation at term: a randomised 
multicentre trial. The Lancet 2000, 356(9239):1375-1383.

29. Rietberg CCT, Elferink-Stinkens PM, Visser GH: The effect of the Term Breech Trial on medical intervention behaviour and neonatal outcome in The Netherlands: an analysis of 35,453 term breech infants. BJOG 2005, 112(2):205-209.

30. Assefa F, Girma W, Woldie M, Getachew B: Birth outcomes of singleton term breech deliveries in Jimma University Medical Center, Southwest Ethiopia. BMC Res Notes 2019, 12(1):428. 